\title{
Plasma properties downstream of a low-power Hall thruster
}

\author{
Brian E. Beal ${ }^{\text {a) }}$ and Alec D. Gallimore \\ Plasmadynamics and Electric Propulsion Laboratory, Department of Aerospace Engineering, \\ The University of Michigan, College of Engineering, Ann Arbor, Michigan 48109 \\ William A. Hargus, Jr. \\ Air Force Research Laboratories, Edwards Air Force Base, Edwards, California 93524
}

(Received 16 May 2005; accepted 9 November 2005; published online 12 December 2005)

Triple Langmuir probes and emissive probes were used to measure the electron number density, electron temperature, and plasma potential downstream of a low-power Hall thruster. The results show a polytropic relation between electron temperature and electron number density throughout the sampled region. Over a large fraction of the plume, the plasma potential obeys the predictions of ambipolar expansion. Near the thruster centerline, however, observations show larger gradients of plasma potential than can be accounted for by this means. Radial profiles of plasma potential in the very-near-field plume are shown to contain large gradients that correspond in location to the boundaries of a visually intense plasma region. (C) 2005 American Institute of Physics.

[DOI: $10.1063 / 1.2145097$ ]

\section{INTRODUCTION}

Many future space missions will use electric propulsion systems for spacecraft station keeping, rephasing, and orbit topping applications. One device particularly well suited to many near-Earth missions is the Hall thruster due to its combination of high reliability and high thrust density at moderately high specific impulses. The Hall thruster is an annular device in which a propellant, usually xenon, is ionized and then accelerated by electrostatic forces to create propulsive thrust. In this type of device, electrons from a thermionically emitting hollow cathode proceed upstream toward a positively biased anode where they ionize the injected propellant. A radial magnetic field imposed by an electromagnetic circuit impedes the motion of electrons toward the anode. The magnetic-field strength is such that the electron gyroradius is much smaller than the characteristic dimensions of the device, while the ion gyroradius is much larger. This arrangement facilitates a strong axial electric field within the plasma and provides for acceleration of the positively charged xenon ions. Upon exiting the device, the ion beam is neutralized by electrons from the hollow cathode, thus maintaining quasineutrality within the plasma plume. The crossed electric and magnetic fields cause electrons in the discharge chamber to drift azimuthally, thereby creating a closed-drift electron Hall current from which this type of thruster derives its name. Figure 1 shows a cross-sectional view of a typical Hall thruster.

One thruster of particular interest to near-term missions is the Busek BHT-200 $200 \mathrm{~W}$ class device, which is shown in Fig. 2. A comprehensive effort to characterize the plume properties of this engine has been undertaken. ${ }^{1-3}$ The plasma density, electron temperature, and plasma potential profiles in the plume of the BHT-200 were measured using a combination of electrostatic triple Langmuir probes and floating

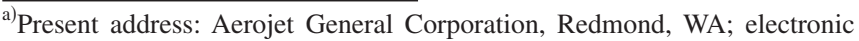
mail: brian.beal@rocket.com
}

emissive probes. This article presents brief descriptions of the vacuum chamber, Hall thruster, and diagnostic techniques. The measured plasma properties are then discussed in detail with particular emphasis on processes occurring near the thruster centerline where the axial gradient of plasma potential is larger than can be accounted for by ambipolar diffusion.

\section{EXPERIMENTAL APPARATUS}

\section{A. Thruster}

The Busek BHT-200 Hall thruster serves as the test article for these experiments and is shown in Fig. 2, which also shows the coordinate system referred to throughout this article. An earlier version of this thruster was reported to operate at an anode efficiency of $42 \%$ and a specific impulse of $1300 \mathrm{~s}$ while providing $12.4 \mathrm{mN}$ of thrust at the nominal operating condition. ${ }^{4}$ The thruster discharge channel has a mean diameter of $21 \mathrm{~mm}$ and is approximately $8 \mathrm{~mm}$ in width. A Busek 3.2-mm-diam hollow cathode is used as an electron source for ionization of the xenon propellant and neutralization of the resulting ion beam. Throughout the experiments described here, the thruster was operated at nominal discharge conditions of $250 \mathrm{~V}$ and $0.80 \mathrm{~A}$ on anode and cathode propellant mass flow rates of 0.83 and $0.10 \mathrm{mg} / \mathrm{s}$, respectively.

\section{B. Vacuum facility}

All experiments discussed in this article were performed in Chamber 6 at the Air Force Research Laboratory (AFRL). Chamber 6 is a cryogenically pumped, 1.8-m-long $\times 3.0$-m-diam cylindrical, stainless-steel vacuum chamber, which is described in detail elsewhere. ${ }^{5}$ This chamber provided a base pressure of $3.2 \times 10^{-7}$ Torr as measured by a cold cathode gauge. During thruster operation, the pressure in the chamber rose to approximately $5.8 \times 10^{-6}$ Torr corrected for xenon. 


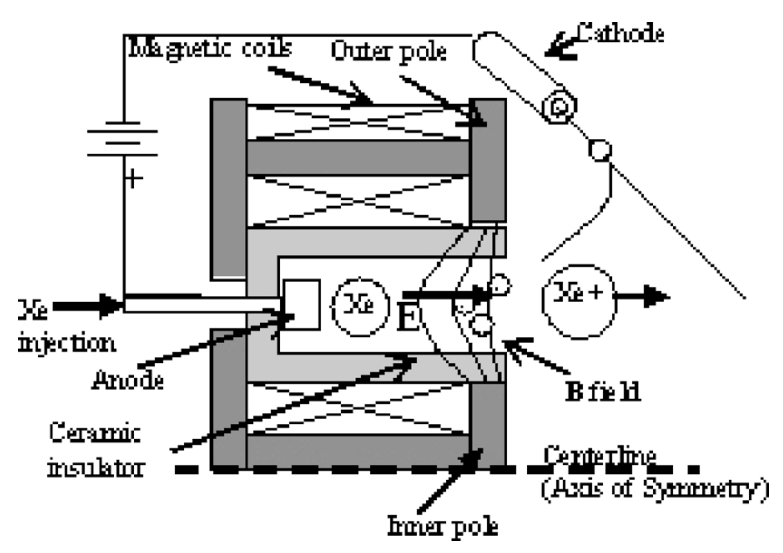

FIG. 1. A sketch of a typical Hall thruster acceleration channel. Note the axis of symmetry.

\section{Triple probe}

The symmetric triple probe, originally developed by Chen and Sekiguchi, ${ }^{6}$ is a convenient plasma diagnostic for collecting large amounts of electron temperature and density data due to the elimination of the voltage sweep required by other electrostatic probes. Additionally, since the probe as a whole floats, the disturbance to the ambient plasma is minimized compared to single Langmuir probes, which draw a net current from the discharge. The triple probes used for this experiment consisted of three tungsten electrodes insulated from each other by an alumina rod. Two separate probes were used. For the larger probe, the diameter of each electrode was $0.50 \mathrm{~mm}$ and the length extending past the end of the alumina was $5.0 \mathrm{~mm}$. A smaller probe consisting of 0.38-mm-diam, 3.8-mm-long electrodes was used in areas of the plume where improved spatial resolution was desired. In

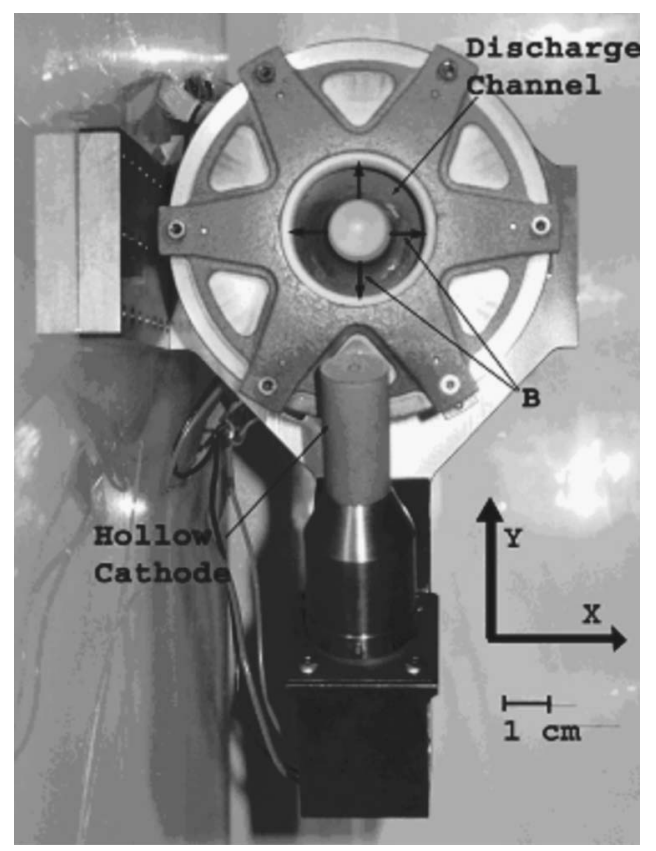

FIG. 2. The BHT-200-X3 low-power Hall thruster and referenced coordinate system. The origin of the coordinate system is located at the intersection of the thruster exit plane and the axis of symmetry. each case, the electrodes were aligned parallel to the thruster axis and spaced approximately two electrode diameters apart. The probes were sized to criteria that allowed the standard assumptions of probe theory to be applied. ${ }^{7}$ Further, it was assumed that the electrodes were sufficiently separated to avoid interaction with each other and that the spatial gradients of plasma properties were sufficiently small to ensure that all three electrodes were exposed to identical plasmas. The limited areas where this last assumption is questionable are discussed in more detail below. Probe dimensions measured before and after the tests described here revealed no changes in probe dimensions as a result of exposure to a streaming plasma.

The method of data analysis used to derive plasma properties from raw probe data has been described in detail elsewhere. ${ }^{8}$ Various previously published error analyses indicate that the absolute uncertainties in the calculated electron temperature and number density for typical triple probes are generally less than $30 \%$ and $60 \%$, respectively. ${ }^{6,9}$ The relative uncertainty between two data points recorded using the same probe is believed to be significantly lower than the absolute uncertainty because many potential sources of error (i.e., uncertainty in probe dimensions, slight asymmetry of the electrodes, etc.) remain constant over the entire spatial region.

\section{Emissive probe}

Plasma potential measurements were conducted using a floating emissive probe similar to the one described by Haas and Gallimore. ${ }^{10}$ The emitting portion of the probe consisted of a loop of 0.13-mm-diam tungsten filament, the ends of which were inserted into double bore alumina tubing along with $0.51-\mathrm{mm}$-diam molybdenum wire leads. Short lengths of tungsten wire were inserted into the alumina tube to ensure contact between the emitting filament and molybdenum leads. The diameter of the emitting filament loop was approximately $3 \mathrm{~mm}$ and the normal to the plane of the loop formed by the emitting filament was oriented in the $X$ direction shown in Fig. 2.

The emissive probe is a widely used plasma diagnostic whose operation is based on the premise that a thermionically emitting filament in a low-temperature plasma will approach the local plasma potential when its emitted electron current is sufficient to neutralize the plasma sheath. ${ }^{11}$ In actuality, the floating potential of the emissive probe remains slightly below the true plasma potential due to space-charge saturation of the sheath. For heavy ions, such as xenon, Ye and Takamura have shown that the difference between the probe potential and the true plasma potential can be as much as 1.03 times the local electron temperature (in eV). ${ }^{12}$ In the far field of the Hall thruster $(Z \geqslant 50 \mathrm{~mm})$, the error induced by this mechanism is less than $3 \mathrm{~V}$, while in the very near field the absolute error may be as much as $5 \mathrm{~V}$.

For this experiment, the current necessary to heat the probe was provided by a programmable power supply with floating outputs. At each location in the plume, the current was steadily increased and the potential with respect to ground at the negative terminal of the power supply was 


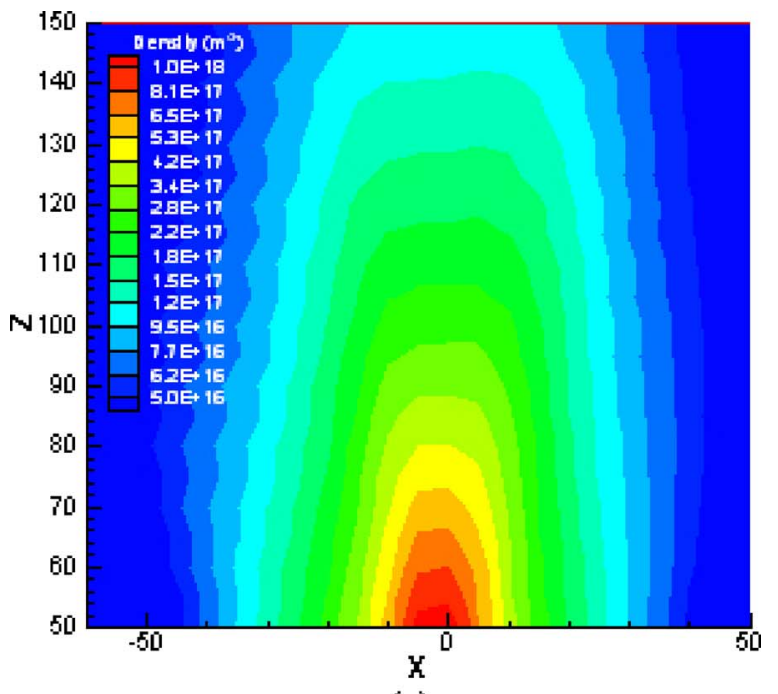

(a)

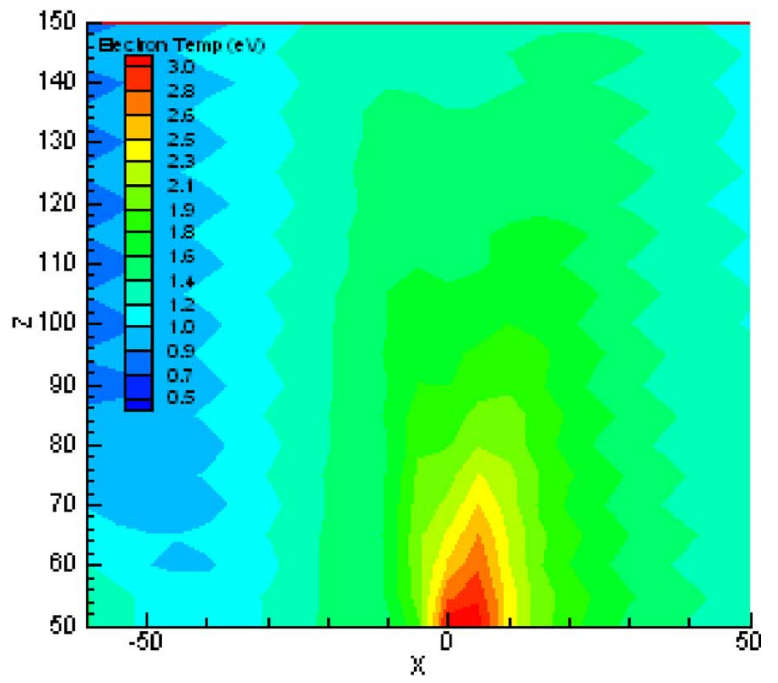

(b)

FIG. 3. (Color online). Electron number density (a) and electron temperature (b) measured in the far-field thruster plume using the large triple probe.

recorded. This method allowed for verification of a welldefined plateau in the voltage-current trace indicating saturation of the plasma sheath. Considering that the voltage drop across the emitting filament never exceeded $6 \mathrm{~V}$, the potential was measured at the negative terminal of the probe, and the electron temperature over the majority of the plume was less than $3 \mathrm{eV}$, the absolute uncertainty in the plasma potential measurements is estimated to be -3 and $+8 \mathrm{~V}$. The relative uncertainty between data points obtained using the same probe is believed to be significantly smaller than this value because the main source of uncertainty, the $\sim 5 \mathrm{~V}$ potential difference across the emitting filament, remained nearly constant over the entire sampled range. The relative uncertainty between data points is therefore conservatively estimated to be $\pm 2.0 \mathrm{~V}$ in the far field $(Z>50 \mathrm{~mm})$ and $\pm 4.0 \mathrm{~V}$ in the near field $(Z<50 \mathrm{~mm})$. This uncertainty is dominated by variations in electron temperature that can influence the small potential drop across the sheath surrounding the emitting filament.

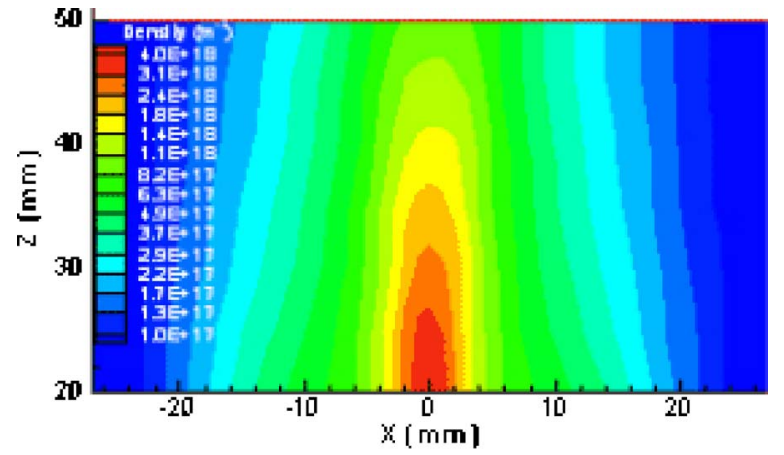

(a)

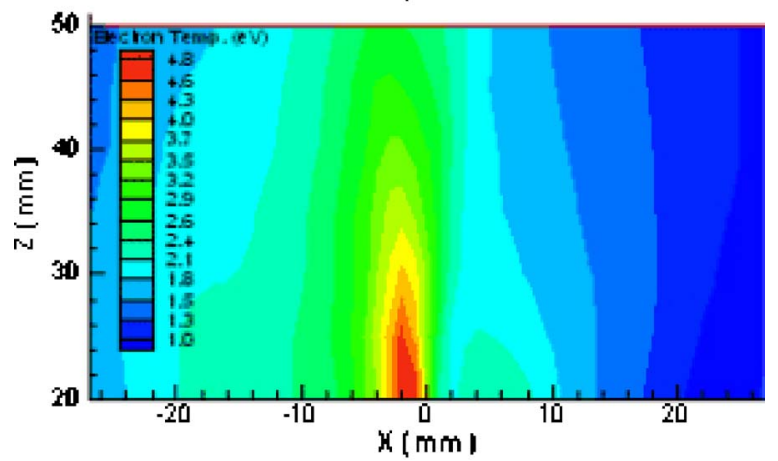

(b)

FIG. 4. (Color online). Near-field electron number density (a) and electron temperature (b) measured with the large triple probe. Note that the density contours are distributed exponentially for clarity.

\section{EXPERIMENTAL RESULTS AND ANALYSIS}

\section{A. Triple probe}

The larger triple probe was used to measure the electron number density and electron temperature at $5 \mathrm{~mm}$ intervals in the far-field Hall thruster plume. These data were taken in a plane perpendicular to the thruster face (the $X Z$ plane in the coordinate system shown in Fig. 2) and are presented in Fig. 3 for axial locations $50-150 \mathrm{~mm}$ downstream of the thruster exit plane. Figure 3(a) shows a well-defined jet structure with a peak plasma density greater than $1 \times 10^{18} \mathrm{~m}^{-3}$ along the centerline of the thruster $50 \mathrm{~mm}$ downstream of the exit plane. The density falls off rapidly in both the downstream and transverse directions and is shown to decrease to less than $1.5 \times 10^{17} \mathrm{~m}^{-3}$ on centerline just $150 \mathrm{~mm}$ downstream of the thruster. The electron temperature distribution, displayed in Fig. 3(b), shows a similar structure to the plasma density plot with maximum temperatures occurring on centerline and decreasing in both the axial and transverse directions. The electron temperature varies between 1 and $2 \mathrm{eV}$ over the majority of the displayed area and increases to nearly $3 \mathrm{eV}$ along the thruster centerline at a distance of $50 \mathrm{~mm}$.

In order to study the processes that occur near the centerline of the Hall thruster plume, measurements were taken in the near field using the smaller triple probe. Figure 4 shows electron number density and electron temperature data obtained at intervals of $1 \mathrm{~mm}$ in the $X$ direction and $5 \mathrm{~mm}$ in the $Z$ direction. These plots show several interesting features. The high-density core seen in the far-field data is very pro- 


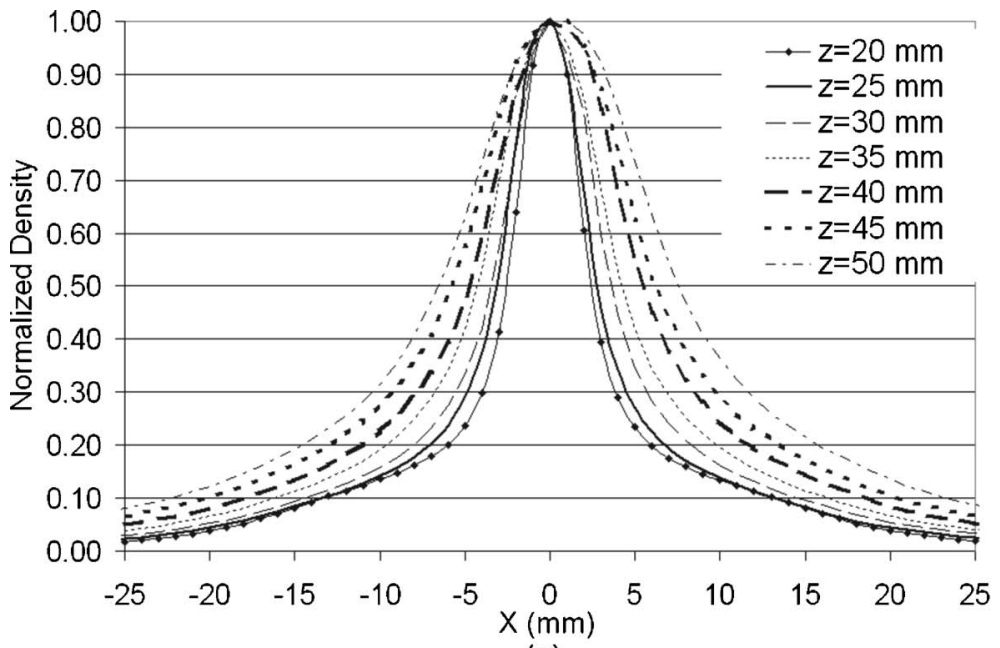

(a)

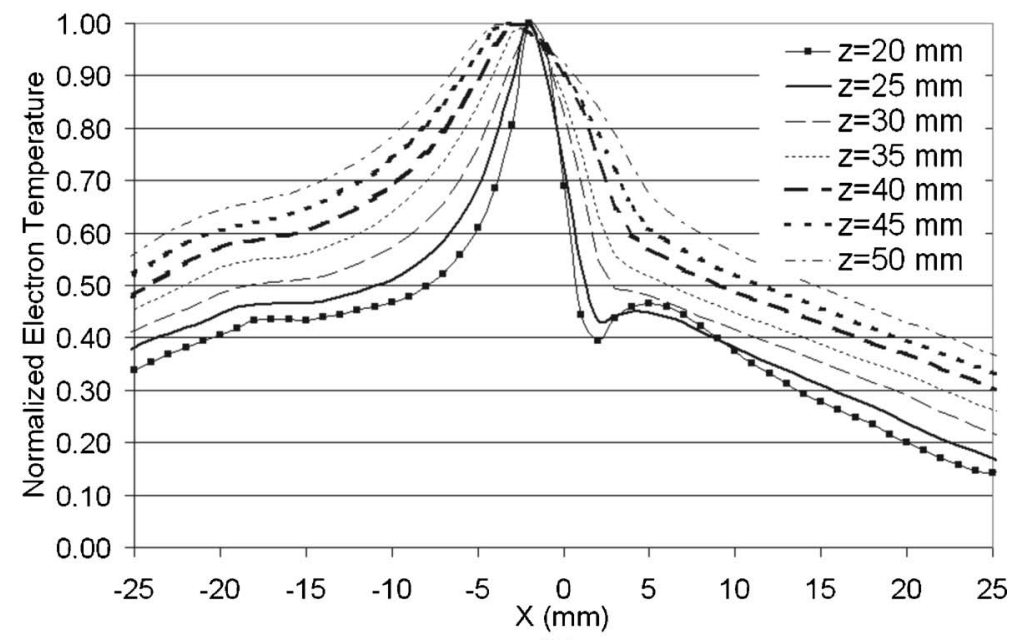

(b)
FIG. 5. Normalized electron density (a) and temperature (b) measured with the small triple probe. Note the short distances over which large changes occur near the thruster centerline. nounced in the near-field measurements. Likewise, the highelectron temperature core alluded to previously is the dominant feature of Fig. 4(b). In the very near field, the electron temperature was found to exceed $5 \mathrm{eV}$ while the peak plasma density reached approximately $4 \times 10^{18} \mathrm{~m}^{-3}$.

Although the data presented in Fig. 4 are believed to be accurate, a word of caution is necessary regarding the level of uncertainty in the very-near-field data. As mentioned previously, a fundamental assumption in the derivation of the governing triple probe equations is that all three electrodes comprising the triple probe are exposed to identical plasma properties. ${ }^{6}$ Near the thruster centerline and close to the discharge channel exit plane, the measured electron temperature and number density change over such a short distance that this assumption is not justified for this region of the plume. Figures 5(a) and 5(b) show normalized traces of the electron number density and electron temperature, respectively, measured at various locations downstream of the thruster face using the small triple probe. The data in each curve are normalized by the maximum value recorded at the given axial (Z) location. These traces show that both the electron temperature and number density appear to change by as much as $20 \%$ over a distance of just $1 \mathrm{~mm}$ near the plume centerline at the upstream end of the sampled region. Considering that the electrodes of the small probe are separated by approximately $2 \mathrm{~mm}$, it can be said that the identical plasma assumption becomes tenuous near the thruster centerline. The absolute uncertainty in the plasma parameters is therefore greater in this region than throughout the remainder of the plume, although the trends and approximate scale of the data presented here are still believed to be representative.

\section{B. Emissive probe}

Figure 6 shows plasma potential measurements taken in the $X Z$ plane of the thruster at axial locations ranging from 50 to $150 \mathrm{~mm}$ downstream of the exit plane. These data were recorded at $5 \mathrm{~mm}$ intervals in both the $X$ and $Z$ directions. Like the density and electron temperature, the plasma potential is highest near the thruster centerline and falls off rapidly in the radial, or $X$, direction. At $50 \mathrm{~mm}$ downstream, the plasma potential reaches a maximum of approximately $20 \mathrm{~V}$ along centerline and falls to less than $5 \mathrm{~V}$ at the boundaries of the sampled region. By $150 \mathrm{~mm}$ downstream, the peak plasma potential decreases to less than $10 \mathrm{~V}$ with respect to the grounded vacuum chamber.

Near-field plasma potential measurements recorded 20-40 $\mathrm{mm}$ downstream of the thruster exit plane are pre- 


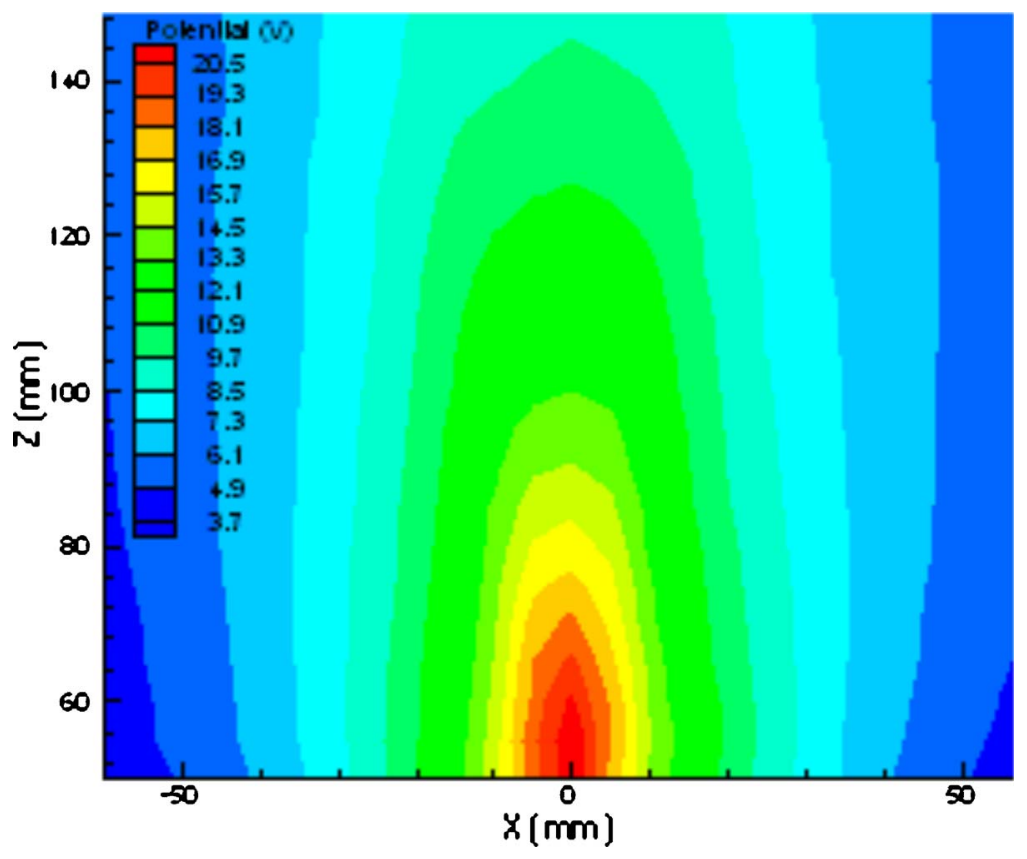

FIG. 6. (Color online). Plasma potentials measured in the thruster plume using a floating emissive probe.

sented in Fig. 7(a). In this figure, the plasma potential can be seen to increase sharply near the thruster centerline, especially at short distances downstream of the exit plane. The data taken $20 \mathrm{~mm}$ downstream, for example, show an increase of roughly $8 \mathrm{~V}$ over a radial distance of approximately $2 \mathrm{~mm}$. Assuming that this structure is axisymmetric, the physical size of the probe must again be taken into consideration. It should be noted that the diameter of the emitting filament loop is approximately $3 \mathrm{~mm}$ and hence is only marginally smaller than the width of the observed highpotential core. However, this does not qualitatively change the result that a region of high plasma potential appears along the thruster centerline and that the rise from the value in the surrounding plasma occurs over a relatively short distance. It does, on the other hand, imply that the apparent width of the high-potential region should be considered a rough approximation since slight misalignment of the probe in the $Y$ direction could cause the core to appear narrower than it would appear to a perfectly aligned, infinitely small probe. For the purposes of comparison, plasma potentials inferred from triple probe data according to Eq. (1) (Ref. 6) are shown in Fig. 7(b), where a high-potential core similar to that shown in Fig. 7(a) can be seen. In Eq. (1), $\phi$ represents plasma potential, $V_{f}$ represents floating potential, and $m_{e}$ and $m_{i}$ represent electron and ion masses, respectively:

$$
V_{p}=V_{f}+\left|\left(\frac{k_{b} T_{e}}{e}\right) \ln \left[\left(\frac{2 \pi m_{e}}{m_{i}}\right)^{1 / 2} \exp \left(-\frac{1}{2}\right)\right]\right| \text {. }
$$

\section{ANALYSIS AND DISCUSSION}

One of the most critical aspects associated with integrating a Hall thruster onto a spacecraft is the need to understand the spatial evolution of the plasma plume properties such that their effects on critical components can be quantified and predicted. In light of this requirement, there are three interesting aspects of the data presented above that merit further discussion: the relationship between the electron temperature and plasma density, the rate of change of plasma potential in the far field, and the unexpected jump in plasma potential that appears near the thruster centerline in the very near field.

\section{A. Equation of state}

Figure 8 shows the correlation between the measured electron temperature and number density in the near-field plume. Data are shown for axial locations of 20, 30, and $40 \mathrm{~mm}$ downstream of the thruster exit plane. Also shown in Fig. 8 is a fit to the empirical data of the form given by Eq. (2), which can be derived from a simple thermodynamic equation of state. ${ }^{13}$ In Eq. (2), $C$ is an arbitrary constant and $\gamma$ represents the effective polytropic index. As shown in Fig. 8 , a value of approximately $\gamma=1.3$ gives the best fit to the experimental data. The fact that $\gamma=1.3$ yields the best fit is interesting because it falls approximately halfway between the two limiting values that one could make reasonable arguments to expect a priori: $\gamma=1$ for isothermal electrons and $\gamma=1.67$ for adiabatic compression of a cold monatomic fluid. That the observed value falls between these limits suggests that the expansion of the plume occurs too slowly (and with too frequent collisions) for the electrons to remain strictly isothermal, but too quickly for the purely adiabatic value to hold. In other words, the time scale associated with the plume expansion appears to be somewhat less than the time required for the plasma to come into complete thermodynamic equilibrium:

$$
T_{e}=C n_{e}^{\gamma-1}
$$

\section{B. Axial plasma potential gradients}

The second interesting aspect of the data presented in the previous section is the evolution of the plasma potential in the thruster plume, which can be studied in the context of the steady-state generalized Ohm's law given by Eq. (3). ${ }^{14,15}$ In 


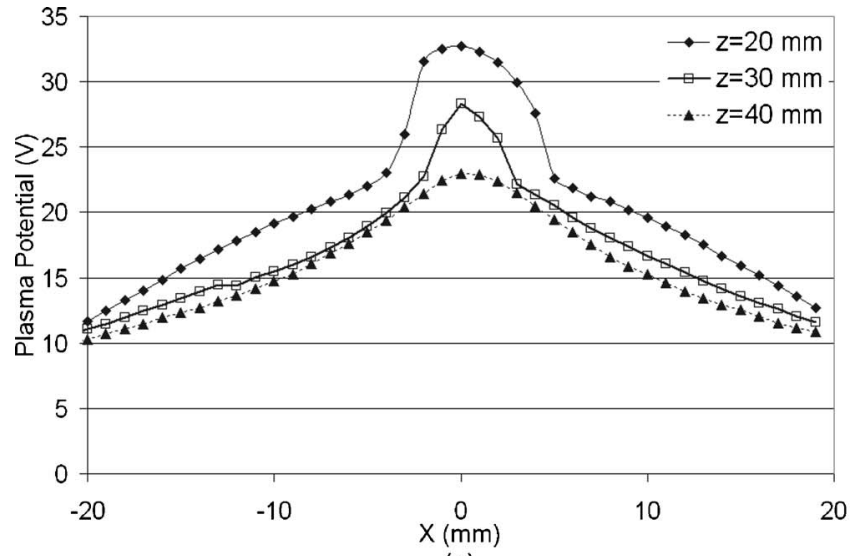

(a)

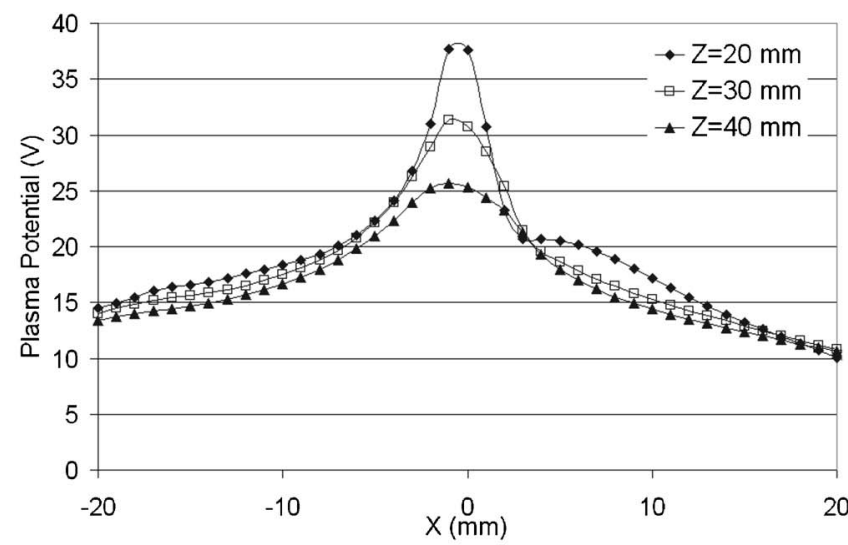

(b)

FIG. 7. Radial profiles of plasma potential measured in the very near-field plume using (a) an emissive probe and (b) a triple probe. Note the large gradients visible at $Z=20$ and $Z=30 \mathrm{~mm}$.

Eq. (3), $E$ is the electric field, $v$ is the plasma drift velocity, $B$ is the magnetic-field strength, $\eta$ represents the plasma resistivity, and $p_{e}$ is the electron pressure given by Eq. (4). The current density $j$ is the sum of contributions from ion and electron components as given by Eq. (5), where quasineutrality has been assumed and $u_{i}$ and $u_{e}$ represent ion and electron axial velocities, respectively. ${ }^{15}$ In the use of Eq. (3) rather than the electron fluid momentum equation, it has been implicitly assumed that the electron-neutral collision frequency is small compared to the electron-ion collision frequency and that the xenon neutral velocity is small with respect to the electron fluid velocity. Although the magnetic field plays a critical role in the internal plasma structure of the Hall thruster, it has been shown that beyond approximately $50 \mathrm{~mm}$ downstream of the exit plane, the magnetic-field strength is less than $5 \mathrm{G}(0.5 \mathrm{mT})$ and the ratio of kinetic pressure to magnetic pressure is greater than $4 .^{5}$ Further, in this region of the plume, the magnetic-flux lines have been shown to be nearly axial in direction such that the radial field strength approaches zero. ${ }^{5}$ For these reasons, the terms involving the magnetic-field strength in Eq. (3) can be omitted when studying the evolution of plasma parameters in the axial $(Z)$ direction. The result can then be written in the differential form of Eq. (6):

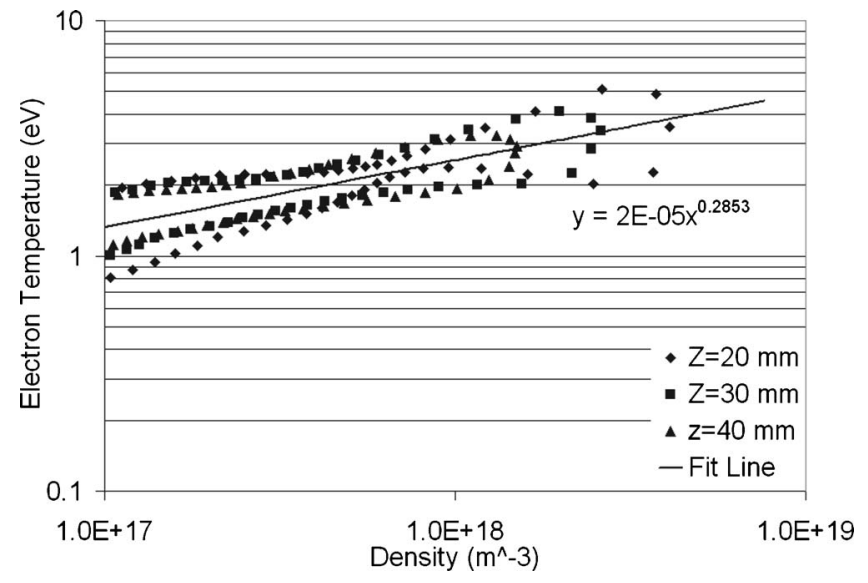

FIG. 8. The correlation between electron temperature and plasma density in the plume of a low-power Hall thruster.

$$
\begin{aligned}
& \mathbf{E}+\mathbf{v} \times \mathbf{B}=\eta \mathbf{j}+\frac{1}{n_{e} e}\left(\mathbf{j} \times \mathbf{B}-\nabla p_{e}\right), \\
& p_{e}=n_{e} k_{b} T_{e}, \\
& j=n_{e} e\left(u_{i}-u_{e}\right), \\
& d \phi=-\eta j d z+\frac{1}{n_{e} e} d p_{e} .
\end{aligned}
$$

The plume of a Hall thruster is generally considered to be a currentless region where electrons migrate downstream at the same density and velocity as the beam ions. In this case, the first term on the right-hand side of Eq. (6) vanishes and the plasma potential can be approximated to be that which exactly cancels the electron pressure gradient. Figure 9 shows plasma potential data recorded at various radial $(X)$ locations from the plume centerline as a function of axial distance $(Z)$ downstream of the exit plane. These data are compared to predictions made according to Eq. (6) (with the $\eta j$ term neglected) by starting with a measured value of plasma potential at the upstream end of the sampled range as a boundary condition. A simple marching algorithm is then used to calculate the axial variation of plasma potential based on the measured plasma density and electron temperature. Predictions made in this manner are labeled "Predicted $(j$ $=0)$ " in Fig. 9 .

The measured plasma potential along the thruster centerline declines more rapidly than can be explained by purely ambipolar effects, as evidenced by the consistent overprediction that results from application of Eq. (6) with $j=0$ in Fig. 9(a). As the radial distance from the thruster centerline is increased, the measured plasma potential and the prediction based on ambipolar expansion begin to agree more closely, and by $X=25 \mathrm{~mm}$ the two values agree to within $0.5 \mathrm{~V}$, which is well within the measurement uncertainty, over the entire axial distance, as shown in Fig. 9(c). The magnitude of the error in the ambipolar prediction method is illustrated by Fig. 10, which shows the overprediction of plasma potential at various radial positions as a percentage of the measured local plasma potential. It is interesting to note that between 

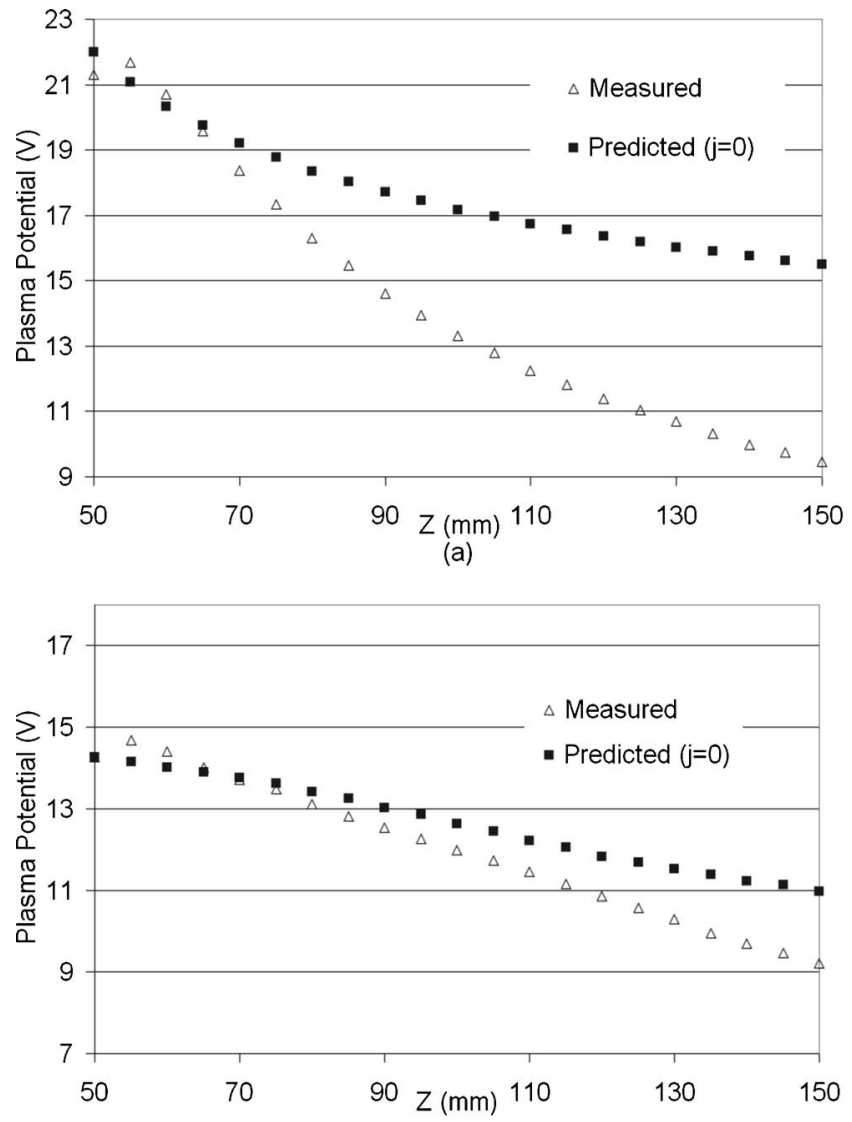

(b)

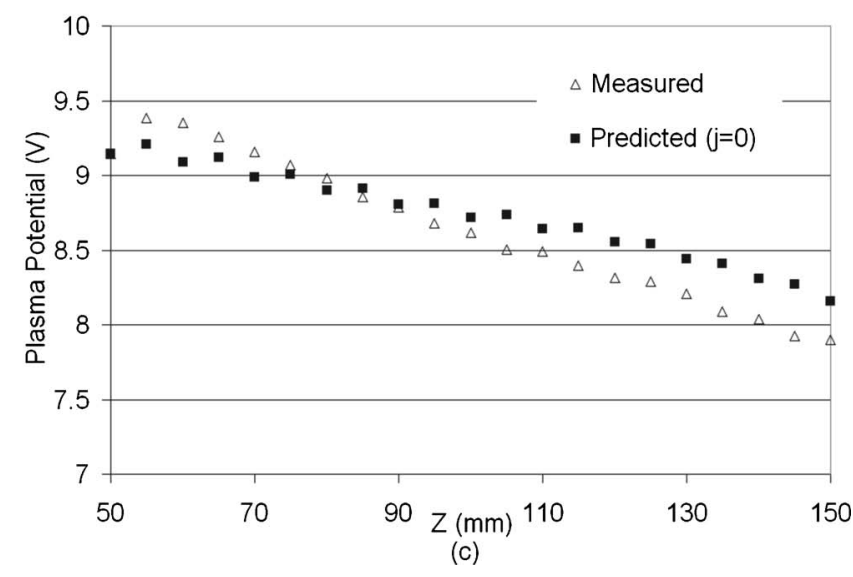

FIG. 9. Measured and predicted values of plasma potential as a function of axial location for radial distances from the thruster centerline of (a) $0 \mathrm{~mm}$, (b) $10 \mathrm{~mm}$, and (c) $25 \mathrm{~mm}$.

the thruster centerline and the discharge channel centerline $(0 \leqslant X \leqslant 10 \mathrm{~mm})$, the overprediction percentage tends to increase with downstream distance, and the magnitude of the overprediction is largest near the thruster centerline. Outside of this narrow central region (i.e., for $X>10 \mathrm{~mm}$ ), the measured and predicted plasma potential values agree to within approximately $\pm 10 \%$ and there is no apparent trend toward increasing or decreasing error with axial distance. The source of the deviation between measured and predicted values near the thruster centerline remains unexplained.

\section{Large radial potential gradients}

The final notable features of the data presented above are the large radial gradients of plasma potential that are apparent near the thruster centerline in the very-near-field plume. These features are readily visible in Fig. 7 at axial distances of 20 and $30 \mathrm{~mm}$ downstream of the thruster exit plane, but are not apparent in data recorded further downstream. Caution must be used when assessing the data in these regions due to the aforementioned similarity between the physical size of the probe and the apparent dimensions of the highpotential region. Despite this limitation, the relatively large change in measured plasma potential and the short distance over which it occurs are striking. Also notable is the apparent correlation between the location of this large potential gradient and the highly luminescent region of the plume that can be seen directly downstream of each thruster in Fig. 11, which shows four identical engines operating simultaneously. The occurrence of such a distinct, bounded region of the plume is widely referred to as operation in "jet mode" and is characteristic of many high-performance thrusters. ${ }^{4}$ Although the "jet" region in the BHT-200 appears simply as a bright plasma core, in many larger thrusters it appears as a cone-shaped area with highly luminescent boundaries. ${ }^{5}$

One hypothesis that has been put forth to explain the occurrence of a well-defined, cone-shaped jet is the development of collisionless shock waves due to the convergence of the ion beam from the annular discharge channel. ${ }^{4}$ It has been shown that both the plasma properties in the plume and the speed of the accelerated ions are consistent with the formation of magnetosonic shock waves. ${ }^{5}$ To the authors' knowledge, however, none of the Hall thruster plume data published to date show conclusive evidence proving or disproving the existence of a shock. The plasma potential profiles presented in Fig. 7 show a nearly discontinuous rise that may support the shock hypothesis. Electron number density and temperature data, on the other hand, show no such evidence. These data then remain inconclusive as to whether or not the appearance of collisionless shocks is responsible for the well-defined structures observed downstream of most high-performance Hall thrusters.

Finally, due to the uncertainty in whether or not collisionless shocks exist in the thruster plume, it is prudent to consider whether or not the observed rise in plasma potential could be caused by other mechanisms. The first mechanism that may account for this observation is the presence of the ion-ion two-stream instability that results as the annular ion beam converges on the thruster centerline. While this phenomenon has been widely reported to be responsible for the formation of collisionless shocks in counterstreaming plasmas, ${ }^{16,17}$ it is entirely possible that such a mechanism may cause sufficient dissipation to lead to a rise in plasma potential without resulting in a true shock. ${ }^{5}$ Second, the rise in plasma potential shown in Fig. 7 could be caused by the formation of an electric double layer separating a highpotential central core from the remainder of the plasma plume. Due to the lack of direct evidence for a shock and the presence of other possible explanations, it must be concluded 


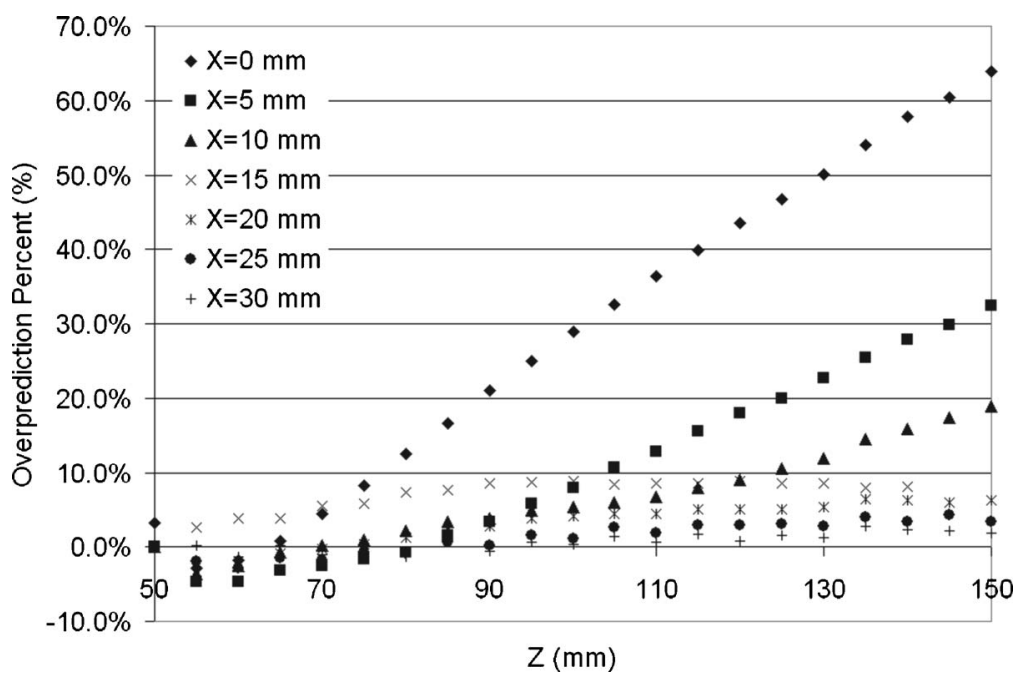

FIG. 10. The overprediction in plasma potential resulting from application of the generalized Ohm's law with the effects of current flow neglected. Note the significant error near the thruster centerline and the good agreement between measurements and predictions at radii greater than $10 \mathrm{~mm}$ from the thruster axis.

that the occurrence of collisionless shocks in the plume is a feasible, but perhaps unnecessary, explanation for the observed phenomena.

\section{CONCLUSIONS}

Plasma number density, electron temperature, and plasma potential measurements taken in the plume of a lowpower Hall-effect thruster show these properties to obey the relations of ambipolar expansion over a large spatial region. Near the thruster centerline, however, the gradient of plasma potential in the axial direction is larger than can be accounted for by ambipolar expansion. Additionally, large radial gradients of plasma potential observed in the very-near-field plume coincide with the boundaries of a visible plasma "jet" and may indicate the occurrence of nonlinear phenomena such as collisionless shocks near the thruster centerline. The evolution of plasma parameters in this region remains partially unexplained and represents a potentially fruitful area for future study.

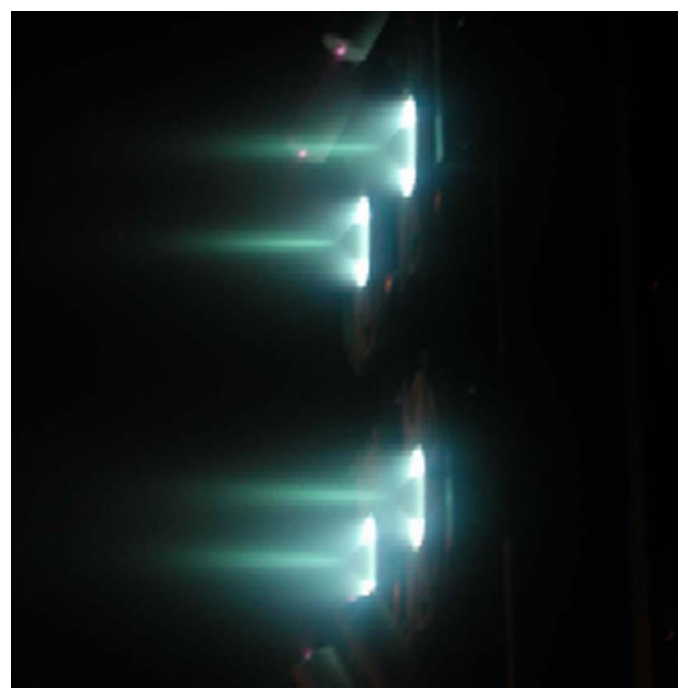

FIG. 11. (Color online). Four low-power Hall thrusters in operation. Note the bright plasma core visible along the centerline of each device.

\section{ACKNOWLEDGMENTS}

The authors wish to thank Dr. Mitat Birkan of the Air Force Office of Scientific Research for sponsoring this project. In addition, the authors would like to thank Professor Iain Boyd of the University of Michigan for useful discussions during the preparation of this manuscript and for sharing simulation results.

A portion of this work was performed under the auspices of AFOSR Grant No. F49620-02-1-0051.

${ }^{1}$ B. E. Beal, A. D. Gallimore, and W. A. Hargus, Proceedings of the 2002 AIAA Joint Propulsion Conference (American Institute of Aeronautics and Astronautics, Washington, DC, 2002), AIAA Paper No. 2002-4251.

${ }^{2}$ W. A. Hargus and G. Reed, Proceedings of the 2002 AIAA Joint Propulsion Conference (American Institute of Aeronautics and Astronautics, Washington, DC, 2002), AIAA Paper No. 2002-3678.

${ }^{3}$ B. E. Beal and A. D. Gallimore, Proceedings of the 2003 International Electric Propulsion Conference (Electric Rocket Propulsion Society, Santa Fe, NM, 2001), IEPC Paper No. 2003-0035.

${ }^{4}$ V. Hruby, J. Monheiser, B. Pote, P. Rostler, J. Kolencik, and C. Freeman, Proceedings of the 1999 AIAA Plasmadynamics and Lasers Conference (American Institute of Aeronautics and Astronautics, Washington, DC, 1999), AIAA Paper No. 99-3534.

${ }^{5}$ B. E. Beal, Ph.D dissertation, University of Michigan, 2004.

${ }^{6}$ S. Chen and T. Sekiguchi, J. Appl. Phys. 36, 2363 (1965).

${ }^{7}$ L. Schott, in Plasma Diagnostics, edited by W. Lochte-Holtgreven (AIP, Woodbury, NY, 1995), Chap. 11, pp. 668-725.

${ }^{8}$ B. E. Beal, A. D. Gallimore, J. M. Haas, and W. A. Hargus, Jr., J. Propul. Power 20, 985 (2004).

${ }^{9}$ D. L. Tilley, A. D. Gallimore, A. J. Kelly, and R. G. Jahn, Rev. Sci. Instrum. 65, 678 (1994).

${ }^{10}$ J. M. Haas and A. D. Gallimore, Phys. Plasmas 8, 652 (2001).

${ }^{11}$ R. F. Kemp and J. M. Sellen, Jr., Rev. Sci. Instrum. 37, 455 (1966).

${ }^{12}$ M. Y. Ye and S. Takamura, Phys. Plasmas 7, 3457 (2000).

${ }^{13}$ D. R. Nicholson, Introduction to Plasma Theory (Wiley, New York, 1983), Chap. 7.

${ }^{14}$ F. F. Chen, Introduction to Plasma Physics and Controlled Fusion (Plenum, New York, 1984).

${ }^{15}$ R. J. Goldston and P. H. Rutherford, Introduction to Plasma Physics (Institute of Physics, Philadelphia, PA, 1997), pp. 116-117.

${ }^{16}$ C. F. Mckee, Phys. Rev. Lett. 24, 990 (1970).

${ }^{17}$ D. A. Tidman and N. A. Krall, Shock Waves in Collisionless Plasmas (Wiley, New York, 1971). 